\title{
Cambios estructurales y funcionales del hipocampo en pacientes con esclerosis múltiple y su relación con procesos de memoria
}

\author{
Álvaro J. Cruz-Gómez, Antonio Belenguer-Benavides, Beatriz Martínez-Bronchal, M. Sol Fittipaldi-Márquez, \\ Cristina Forn
}

Departamento de Psicología Básica, Clínica y Psicobiología; Facultad de Ciencias de la Salud; Universitat Jaume I (A.J. Cruz-Gómez, B. MartínezBronchal, M.S. Fittipaldi-Márquez, C. Forn). Sección de Neurología; Hospital General de Castellón (A. Belenguer-Benavides). Castellón de la Plana, España.

Correspondencia:

Dr. Álvaro Javier Cruz Gómez. Departamento de Psicología Básica, Clínica y Psicobiología. Facultad de Ciencias de la Salud. Universita Jaume I. Campus Riu Sec. Edificio de Investigación II. E-12071 Castellón.

E-mail: acruz@.uji.es

Financiación:

Subvención concedida por la Universitat Jaume I (P1.1B2014-05)

Aceptado tras revisión externa: 23.10.15

Cómo citar este artículo: Cruz-Gómez AJ, BelenquerBenavides A, Martínez-Bronchal B Fittipaldi-Márquez MS, Forn C. Cambios estructurales y funcionales del hipocampo en pacientes con esclerosis múltiple y su relación con procesos de memoria. Rev Neurol 2016; 62: 6-12.

(c) 2016 Revista de Neurología

Introducción. Una de las alteraciones cognitivas más prevalentes en los pacientes con esclerosis múltiple son los problemas de codificación de la información (tanto verbal como visual), proceso relacionado con el hipocampo.

Objetivo. Estudiar la relación entre los procesos de aprendizaje y retención de la información a largo plazo con el volumen hipocampal y la conectividad funcional (CF) en pacientes con esclerosis múltiple comparados con un grupo control.

Sujetos y métodos. Ambos grupos fueron evaluados con la batería neuropsicológica breve, que incluye pruebas de memoria verbal y visual. Se realizó el estudio de volumetría de la sustancia gris mediante la técnica de morfometría basada en el vóxel y un estudio de CF de vóxel de semilla centrado en la zona de interés (hipocampo). Se realizaron análisis de asociación entre rendimiento en memoria y cambios volumétricos y de CF.

Resultados. Los resultados mostraron atrofia en la sustancia gris en el hipocampo izquierdo y una menor CF entre el hipocampo izquierdo y el troncoencéfalo, el cerebelo, el giro fusiforme y el giro temporal superior en los pacientes respecto al grupo control. En el grupo de pacientes se observa una correlación positiva entre la sustancia gris en ambos hipocampos y el rendimiento en memoria verbal, así como una correlación positiva entre el rendimiento en memoria visual y la CF entre el hipocampo izquierdo y diversas regiones temporales.

Conclusiones. Los resultados muestran una relación entre el rendimiento de memoria verbal y visual, y cambios estructurales y funcionales en el hipocampo en pacientes con esclerosis múltiple.

Palabras clave. Conectividad funcional. Esclerosis múltiple. Hipocampo. Memoria. Sustancia gris. Volumetría.

\section{Introducción}

La esclerosis múltiple (EM) es una enfermedad autoinmune, desmielinizante y degenerativa del sistema nervioso central con manifestaciones clínicas muy heterogéneas. Destacan problemas sensitivos y motores (que incluyen problemas visuales, del equilibrio y del control de los esfínteres, entre otros), así como alteraciones cognitivas y psiquiátricas $[1,2]$. Diversos trabajos indican que los déficits cognitivos en la EM tienen una prevalencia elevada (afectan a un $43-70 \%$ de la población clínica), se manifiestan en cualquier fase de la enfermedad y afectan a la calidad de vida de estos pacientes [1-3]. De forma más específica, algunos trabajos han sugerido que las alteraciones cognitivas son más frecuentes y graves en pacientes con el subtipo de enfermedad secundario progresivo (EMSP) que en aquellos con subtipo remitente-recurrente (EMRR) [4,5]. El patrón de alteración cognitiva incluye déficits en velocidad del procesamiento de la información, alteraciones en las funciones atencionales y ejecutivas, y, por último, alteraciones en las funciones de memoria [6-9]. En cuanto a los procesos de memoria, son los problemas de aprendizaje y memoria a largo plazo los más destacados, afectando a un 34-56\% de pacientes [7].

En esta enfermedad hay una afectación tanto de la sustancia blanca como de la sustancia gris, pero es la atrofia en la sustancia gris la que mayor relación ha mostrado con el bajo rendimiento cognitivo en la EM [10]. Anatómicamente, los procesos de aprendizaje y retención de la información se relacionan con estructuras diencefálicas, y de forma concreta con el hipocampo [11]. Diversos son los trabajos que han estudiado la reducción del volumen en la sustancia gris en el hipocampo en la EM y su relación con los procesos de aprendizaje, con resultados poco concluyentes. En uno de los primeros estudios [6] se midió el volumen del hipocampo de forma global y segmentada en pacientes con EMRR y EMSP. Ambos grupos de pacientes presentaron una disminución del volumen del hipocampo comparado con el grupo control, y esta disminución fue más evidente en la subregión CA1. Además, tam- 
bién se encontró una correlación entre atrofia y un bajo rendimiento en una prueba de aprendizaje verbal. Otro estudio reciente con una amplia muestra de pacientes con EM [9] confirma estos resultados, y ha hallado de nuevo una relación del rendimiento en memoria verbal y visuoespacial con el volumen en la región CA1. Por otro lado, también existen en la bibliografía estudios que, aunque han confirmado la presencia de atrofia hipocampal en pacientes con EM, no han podido demostrar una correlación significativa con las pruebas de memoria [12].

Trabajos recientes han ido dirigidos a complementar la relación inconcluyente entre el deterioro cognitivo en la EM y el grado de daño estructural cerebral con medidas relacionadas con los cambios en los patrones de conectividad funcional (CF) en estado de reposo [13-15]. Estos estudios se centran en investigar en qué medida están conectadas diferentes regiones cerebrales, y analizan su actividad conjunta o sincronización temporal. Sólo encontramos dos estudios que han examinado los cambios de la CF del hipocampo en relación con el rendimiento cognitivo en pacientes con EM, y ambos trabajos muestran resultados difíciles de conciliar. Por un lado, el estudio desarrollado por Roosendaal et al [13] demuestra que aquellos pacientes con mayor atrofia hipocampal presentan una disminución de la CF de esta región con diferentes áreas cerebrales respecto a los sujetos control, aunque no encuentra relación con el rendimiento en memoria (su muestra de pacientes no presenta alteración neuropsicológica). Por otra parte, Hulst et al [15] analizan una muestra de pacientes con EM con problemas en memoria verbal y visuoespacial y, en esta ocasión, encuentran un aumento de la CF del hipocampo izquierdo con el cingulado posterior derecho respecto al grupo control, que además es capaz de predecir parcialmente un peor rendimiento en tareas de memoria.

Ante esta escasez de trabajos y la dificultad para interpretar los resultados obtenidos, son necesarios más estudios para establecer de forma más consistente cómo se relacionan los cambios estructurales y de CF del hipocampo con el deterioro cognitivo en la EM. Teniendo en cuenta estos antecedentes, los objetivos del presente trabajo fueron: estudiar una posible reducción del volumen de la sustancia gris del hipocampo en un grupo de pacientes con EM en comparación con el grupo control, y su relación con el rendimiento en pruebas de aprendizaje y retención de la información, y estudiar una posible alteración de la CF entre el hipocampo y otras estructuras corticales observada en pacientes con EM en comparación con el grupo control, y su rela- ción con el rendimiento en tareas de aprendizaje y retención de la información.

\section{Sujetos y métodos}

\section{Participantes}

Para el siguiente estudio se seleccionaron 78 pacientes diagnosticados de EM (54 mujeres) tratados en el Servicio de Neurología del Hospital General de Castellón, siguiendo los criterios diagnósticos de McDonald de 2005 [16]. Cincuenta y nueve de los pacientes presentaban EMRR, y 19, EMSP. Fueron excluidos aquellos pacientes que presentaban un brote o recaída en el momento de la exploración o durante los dos últimos meses y que estuvieran en tratamiento con esteroides, o aquellos que presentaran otra enfermedad neurológica además del diagnóstico de EM. Todos los pacientes fueron valorados neurológicamente con la Expanded Disability Status Scale y con la Fatigue Severity Scale [17,1 8]. Los pacientes fueron comparados con un grupo de 19 sujetos sanos (nueve mujeres), sin alteración neurológica o psiquiátrica.

El estudio fue aprobado por los comités de ética del hospital y de la Universitat Jaume I, y todos los sujetos dieron su consentimiento por escrito para participar.

\section{Valoración neuropsicológica}

Todos los participantes fueron valorados con la batería neuropsicológica breve Brief Repeatable Battery of Neuropsycological tests (BRB-N) de Rao et al [19], traducida y baremada en población de habla castellana [20]. Esta batería está formada por tests que evalúan las áreas cognitivas más afectadas en la EM [21]. Con los diferentes subtests de esta batería obtuvimos las puntuaciones $Z$ del rendimiento en memoria verbal, y la puntuación $Z$ del rendimiento en memoria visual (funciones cognitivas de interés para el estudio). La $Z$ verbal (Zv) se obtiene del rendimiento de los tres tests de aprendizaje y memoria a largo plazo verbal de la BRB-N), mientras que la $Z$ visual (Zvi) se obtiene del rendimiento de los dos tests de aprendizaje y memoria visuoespacial a largo plazo. Además, se valoró el cociente intelectual (CI) premórbido, utilizando el subtest de la escala Wechsler de inteligencia para adultos-III de matrices, y se obtuvo información sobre el CI manipulativo. Ninguno de los pacientes manifestó dificultades motoras o visuales durante la realización de los diferentes tests neuropsicológicos. 


\section{Adquisición de neuroimagen}

Mediante un escáner Siemens Avanto 1,5 T se adquirió para cada uno de los participantes una imagen morfométrica 3D sagital $\mathrm{T}_{1}$ (TR: $11 \mathrm{~ms}$; FOV: $256 \times 234 \mathrm{~mm}$; matriz: $256 \times 224$; tamaño de vóxel: $1 \times 1 \times 1 \mathrm{~mm}$; TE: 4,9 ms; número de ecos: 1; flip angle: $\left.15^{\circ}\right)$. De forma adicional, se adquirieron 270 volúmenes para el análisis de resonancia magnética funcional en estado de reposo, gradient-echo $T_{2}{ }^{*}$ weighted echo planar imaging sequence (TR/TE): 2.000/30 ms; matriz: $64 \times 64 \times 30$; tamaño de vóxel: $3,5 \times 3,5 \times 4,02 \mathrm{~mm}$; flip angle: $90^{\circ}$. Para la adquisición de la secuencia en estado de reposo se instruyó a todos los participantes a permanecer inmóviles, manteniendo los ojos cerrados e intentando no dormirse ni pensar en nada en particular.

\section{Preprocesado de las imágenes volumétricas}

En primer lugar, se realizó una segmentación semiautomática de las lesiones de los pacientes en las imágenes $3 \mathrm{D}$ sagitales $\mathrm{T}_{1}$ mediante el programa Jim (v. 5.0, Xinapse Systems, Northants, Reino Unido). Después, con la herramienta lesion filling incluida en el FMRIB Software Library [22], se rellenaron las áreas lesionadas con intensidades de los vóxeles de la sustancia blanca adyacentes para mejorar la segmentación de tejidos posterior. A continuación, las imágenes 3D 'rellenadas' fueron reorientadas y preprocesadas con la herramienta VBM8 del programa Statistical Parametrical Mapping (SPM v. 8). Brevemente, el preprocesado incluyó una segmentación de las imágenes en diferentes tejidos, el registro a un template estándar, una normalización, una modulación y un suavizado FWHM de $8 \mathrm{~mm}$.

\section{Preprocesado de las imágenes}

funcionales en estado de reposo

Las imágenes funcionales fueron preprocesadas mediante la herramienta Data Processing Assistant for Resting-State fMRI Advanced (DPARSFA [23]), siguiendo los pasos recomendados por estos autores. Después, seleccionamos como regiones de interés ambos hipocampos, creando las regiones previamente con el programa wfu_Pickatlas [24] para usarlas posteriormente como semillas en los análisis posteriores. Después, calculamos los mapas de la CF vóxel a vóxel para obtener las redes funcionales evocadas por cada hipocampo. Este método permite estudiar la CF en estado de reposo (mediante la correlación de Pearson) de una región semilla con todos los otros vóxeles del cerebro en cada uno de los participantes. Los mapas individuales de correlación $r$ se normalizaron a mapas $z$ con la transformación $Z$ de Fisher, y después se obtuvieron los mapas de conectividad para cada grupo (sujetos control y pacientes).

\section{Análisis estadístico de segundo nivel}

Atendiendo a los objetivos del estudio, se realizaron los siguientes análisis estadísticos de segundo nivel:

- Prueba $t$ para observar las diferencias entre los dos grupos de participantes (pacientes y controles), tanto en las variables conductuales como en la localización de la atrofia hipocampal y las diferencias de la CF.

- Análisis de regresión lineal para observar una posible relación entre las puntuaciones $\mathrm{Zv}$ y Zvi con la atrofia hipocampal, así como con las alteraciones de $\mathrm{CF}$.

Para el análisis de las diferencias estructurales se utilizó una máscara de la región de interés (hipocampos) y una corrección small volume para comparaciones múltiples a nivel de clúster con una $p<$ 0,001 y covariando por edad y sexo. Para los análisis de la CF, se utilizó una $p<0,001$ corregida a nivel de clúster mediante simulación de Monte Carlo, usando la herramienta Alphasim incluida en el programa REST, y covariando todos los análisis por edad y sexo. El etiquetado de las localizaciones neuroanatómicas correspondientes a las áreas estadísticamente significativas se efectuó con la extensión xjview.

\section{Resultados}

\section{Resultados neuropsicológicos}

Los resultados neuropsicológicos para ambos grupos se presentan en la tabla I. El grupo de pacientes se caracteriza por tener una mayor edad, menos años de escolaridad y un menor CI manipulativo que el grupo control. Presenta además un peor rendimiento neuropsicológico en todas las pruebas cognitivas. De forma específica, más de la mitad de los pacientes $(60,87 \%$ de la muestra) presenta un rendimiento alterado en las tareas que evalúan la memoria.

Cuando comparamos pacientes según el subtipo de su enfermedad, los pacientes con EMSP muestran más edad, más años de evolución de enfermedad y un peor rendimiento en todos los tests cognitivos respecto a los pacientes con EMRR (aunque no se observan diferencias entre los grupos en años de escolaridad ni en CI manipulativo). 


\section{Resultados volumétricos}

Los resultados de volumetría obtenidos muestran que los pacientes con EM presentan atrofia en el hipocampo izquierdo respecto al grupo control, concretamente en dos clústeres (coordenadas del Montreal Neurological Institute, MNI: $-15,-33,4,5 ; t=$ $7,17 ; k=348$; MNI: $-12,-7,5,-19,5 ; t=3,74)$. Además, las correlaciones realizadas muestran una correlación positiva en el grupo de pacientes entre la $\mathrm{Zv}$ y la atrofia en el hipocampo, tanto en el izquierdo (dos clústeres, MNI: $-19,5,-7,5,-19,5 ; r=0,53$; $k=441$; MNI: $-12,-37,5,4,5 ; r=0,49 ; k=126)$ como en el derecho (dos clústeres, MNI: 22,5, -34,5, $-3 ; r=0,52 ; k=204$; MNI: $16,5,-10,5,-18, r=0,48$; $k=206$ ) (Fig. 1).

\section{Resultados de conectividad funcional}

Los resultados muestran que el grupo de pacientes en comparación con el de sujetos sanos presenta disminución de la CF entre el hipocampo izquierdo y diversas regiones cerebrales. Además, se observa una correlación positiva entre las puntuaciones de la Zvi en el grupo de pacientes y la CF entre el hipocampo izquierdo y regiones del lóbulo temporal derecho (Tabla II y Fig. 2).

\section{Discusión}

El presente estudio muestra que existe una relación entre la atrofia de la sustancia gris en el hipocampo en los pacientes con EM, que afecta a su vez a la CF de esta zona con otras regiones corticales. Además, también observamos que estas dos variables (atrofia en la sustancia gris y la CF) influyen en el rendimiento de las pruebas que miden el aprendizaje y la retención a largo plazo.

En este trabajo, utilizando la técnica de morfometría basada en el vóxel optimizada con la herramienta DARTEL, hemos observado cómo los pacientes con EM muestran atrofia en el hipocampo izquierdo respecto al grupo control. Estos resultados son similares a los hallados por otros trabajos previos, donde también se describe la presencia de atrofia hipocampal en pacientes con EM $[6,9,12,13$, 15]. Respecto a la relación entre pérdida de volumen y ejecución en las pruebas de aprendizaje y retención a largo plazo en pacientes, encontramos una correlación entre el volumen de ambos hipocampos y el rendimiento en las pruebas de memoria verbal $(\mathrm{Zv})$. Estos resultados denotan que, en los pacientes con EM, peores puntuaciones en memo-
Tabla I. Datos demográficos, clínicos y neuropsicológicos de los participantes (media \pm desviación estándar).

\begin{tabular}{|c|c|c|c|}
\hline & $\begin{array}{c}\text { Controles } \\
(n=19)\end{array}$ & $\begin{array}{c}\text { Pacientes } \\
(n=78)\end{array}$ & $p$ \\
\hline $\begin{array}{l}\text { Años de evolución } \\
\text { de la enfermedad }\end{array}$ & - & $\begin{array}{c}8,68 \pm 7,35 \\
\text { (rango: } 1-29 \text { ) }\end{array}$ & - \\
\hline Edad media & $\begin{array}{c}31,63 \pm 6,06 \\
\text { (rango: } 22-44 \text { ) }\end{array}$ & $\begin{array}{c}39,1 \pm 8,34 \\
\text { (rango: } 20-56 \text { ) }\end{array}$ & 0,00 \\
\hline $\begin{array}{l}\text { Media de años } \\
\text { de escolaridad }\end{array}$ & $\begin{array}{l}13,89 \pm 2,56 \\
\text { (rango: } 8-16 \text { ) }\end{array}$ & $\begin{array}{c}11,1 \pm 2,91 \\
\text { (rango: } 8-16 \text { ) }\end{array}$ & 0,00 \\
\hline $\begin{array}{l}\text { Expanded Disability } \\
\text { Status Scale }\end{array}$ & - & $\begin{array}{c}2,70 \pm 1,61 \\
\text { (rango: } 0-7 \text { ) }\end{array}$ & - \\
\hline Fatigue Severity Scale & $32,74 \pm 10,71$ & $37,48 \pm 16,41$ & 0,24 \\
\hline $\begin{array}{l}\text { Cociente intelectual } \\
\text { manipulativo }\end{array}$ & $112,11 \pm 13,57$ & $100,26 \pm 10,44$ & 0,00 \\
\hline SDMT & $58,21 \pm 11,50$ & $46,10 \pm 14,30$ & 0,00 \\
\hline PASAT-3 & $47,05 \pm 9,95$ & $31,12 \pm 22,51$ & 0,00 \\
\hline SRT-almacenamiento & $51,47 \pm 12,84$ & $40,64 \pm 14,00$ & 0,00 \\
\hline SRT-recuperación & $41,26 \pm 11,09$ & $30,69 \pm 14,07$ & 0,00 \\
\hline SRT-largo plazo & $9,68 \pm 1,77$ & $7,60 \pm 2,66$ & 0,00 \\
\hline 10/36 SPART & $22,47 \pm 4,50$ & $18,95 \pm 5,71$ & 0,00 \\
\hline 10/36 SPART-largo plazo & $8,05 \pm 2,15$ & $6,58 \pm 2,27$ & 0,01 \\
\hline Word List Generation Test & $22,53 \pm 4,62$ & $19,71 \pm 5,87$ & 0,03 \\
\hline
\end{tabular}

SPART: Spatial Recall Test; PASAT: Paced Auditory Serial Addition Test; SDMT: Symbol Digit Modalities Test; SRT: Selective Reminding Test.

ria verbal se asocian a un mayor grado de daño estructural en el hipocampo.

Como hemos visto, la relación entre cambios estructurales en el hipocampo y el rendimiento en pruebas de aprendizaje y memoria a largo plazo en pacientes con EM es, hasta el momento, contradictoria. Mientras que algunos trabajos no han encontrado correlaciones significativas entre estas variables [12,13], otros sí las han hallado [6,9]. Cabe la posibilidad de que la ausencia de relación entre el volumen hipocampal y el rendimiento en pruebas de memoria en pacientes con EM descrita por algunos estudios $[12,13]$ pueda relacionarse con el empleo de medidas de volumen del hipocampo a nivel global. Así, en uno de los trabajos citados [9], el rendimiento en memoria no correlacionó con el vo- 
Figura 1. a) Diferencias en volumetría entre pacientes y controles: atrofia del hipocampo izquierdo en el grupo de pacientes; b) Correlación en el grupo de pacientes entre la Z verbal (Zv) y la atrofia en la sustancia gris (SG) del hipocampo a nivel bilateral. Para el análisis de las diferencias estructurales del hipocampo se utilizó una corrección small volume para comparaciones múltiples en el clúster, con una $p<0,001$, con covarianza por edad y sexo.

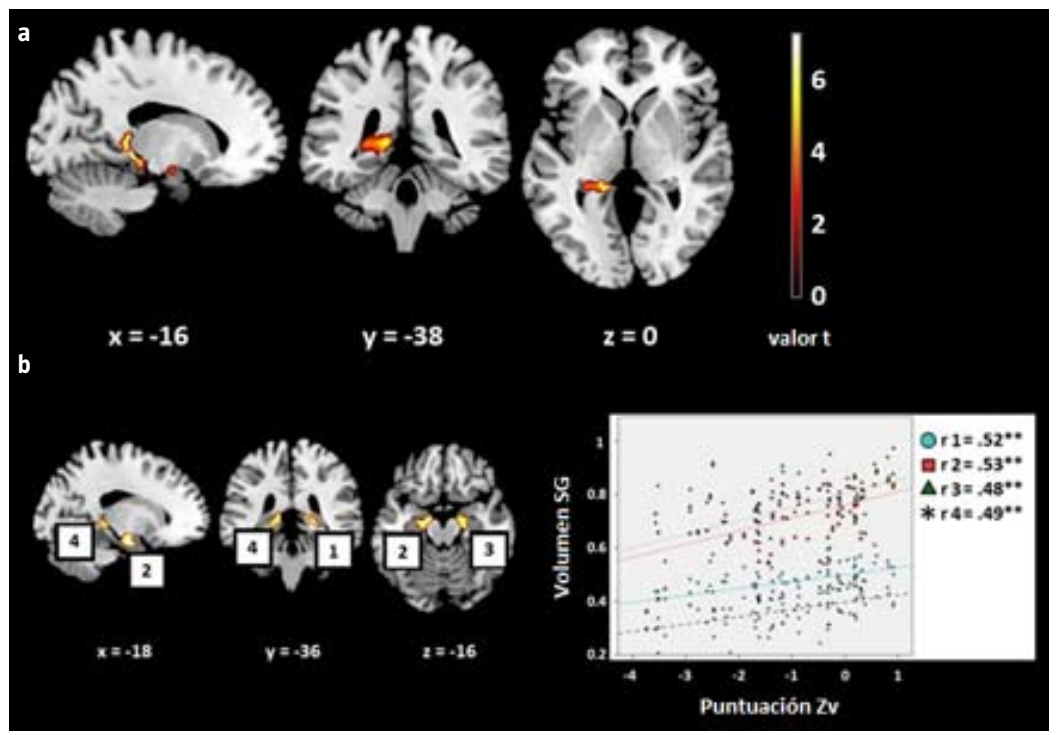

Tabla II. Regiones y coordenadas de las áreas que muestran una menor conectividad funcional (CF) con el hipocampo izquierdo en pacientes con esclerosis múltiple (EM) respecto a controles y áreas cuyo grado de CF correlaciona significativamente con la puntuación en memoria visual (Zvi) en el grupo de pacientes.

\begin{tabular}{|c|c|c|c|c|c|c|}
\hline & & \multirow{2}{*}{$\begin{array}{l}\text { Tamaño } \\
\text { del clúster }\end{array}$} & \multirow{2}{*}{$t$} & \multicolumn{3}{|c|}{ Coordenadas del MNI } \\
\hline & & & & $x(\mathrm{~mm})$ & $y(\mathrm{~mm})$ & $z(\mathrm{~mm})$ \\
\hline \multirow{6}{*}{$\begin{array}{l}\text { Disminución de la } \\
\text { CF del hipocampo } \\
\text { izquierdo en } \\
\text { pacientes con EM } \\
\text { respecto a controles }\end{array}$} & Troncoencéfalo I & \multirow{2}{*}{45} & 4,32 & 0 & -33 & -39 \\
\hline & Troncoencéfalo D & & 4,39 & 9 & -33 & -39 \\
\hline & Cerebelo D & 23 & 4,26 & 0 & -51 & -30 \\
\hline & Giro temporal inferior I & \multirow{2}{*}{22} & 4,25 & -42 & -18 & -33 \\
\hline & Giro fusiforme I & & 3,49 & -36 & -12 & -30 \\
\hline & Cerebelo I & 22 & 3,91 & -27 & -42 & -30 \\
\hline \multirow{4}{*}{$\begin{array}{l}\text { Correlación de la } \\
\text { CF del hipocampo } \\
\text { izquierdo con Zvi } \\
\text { en pacientes con EM }\end{array}$} & Giro temporal superior D & \multirow{4}{*}{31} & \multirow{4}{*}{0,58} & 36 & 6 & -24 \\
\hline & Giro temporal medio D & & & 39 & 6 & -36 \\
\hline & Giro fusiforme D & & & 33 & 0 & -33 \\
\hline & Giro temporal inferior D & & & 36 & 12 & -42 \\
\hline
\end{tabular}

D: derecho; I: izquierdo; MNI: Montreal Neurological Institute. lumen global del hipocampo, aunque sí con el volumen de la subregión CA1. De forma paralela, en nuestro trabajo hallamos una correlación con pruebas de memoria al realizar un análisis volumétrico del hipocampo vóxel a vóxel. Por otro lado, mientras que algunos estudios han empleado técnicas de segmentación automática del hipocampo, otros han utilizado técnicas de segmentación manual, factor que podría contribuir también a la diversidad de resultados hallados [25].

En este trabajo también demostramos una relación entre la atrofia y la CF del hipocampo. En ese sentido, los pacientes con EM muestran una disminución de la CF entre el hipocampo izquierdo (donde se localiza el foco de atrofia) y otras regiones temporales relacionadas con los procesos de memoria declarativa [26,27]. Es difícil comparar estos resultados con estudios anteriores. Como se ha comentado, en el estudio de Roosendaal et al [13] también se relacionó la atrofia del hipocampo con el descenso de la CF en pacientes con EM, aunque no se observó relación con los procesos de memoria (su muestra de pacientes no presentaba alteración en esta área). Así pues, aunque los resultados no son comparables, sí podemos afirmar que ambos trabajos son sugestivos de que la atrofia del hipocampo implica una disminución de la CF entre esta estructura y otras áreas cerebrales.

Nuestros resultados van a favor de la hipótesis de que los aumentos de la CF resultan beneficiosos y podrían constituir un mecanismo de compensación del déficit cognitivo en la EM [14,28], ya que hallamos una correlación positiva que confirma que, a medida que los pacientes muestran una mayor $\mathrm{CF}$ entre el hipocampo izquierdo y zonas temporales derechas (relacionadas con la memoria declarativa/ episódica [27]), rinden mejor en tareas de aprendizaje y memoria de información visuoespacial. Sin embargo, los resultados de Hulst et al [15] van en la línea opuesta, ya que en su muestra de pacientes de EM con alteración en memoria encuentran un aumento de la CF del hipocampo respecto al grupo control, que además correlaciona de forma inversa con el rendimiento en tareas de memoria. Estos autores ponen en entredicho la hipótesis del aumento de la CF como un mecanismo beneficioso y sugieren que representaría más bien una desorganización desadaptativa de la red cerebral implicada en procesos de memoria, que se asociaría a un peor funcionamiento neuropsicológico. Al igual que ocurre con los resultados de volumetría en el hipocampo y su relación con los procesos de memoria, es posible que la diferencia de técnicas utilizadas para objetivar los cambios de la CF del hipocampo, la 
heterogeneidad de las muestras de pacientes empleadas (por ejemplo, con o sin alteración en memoria) o las diferencias en la cuantificación del grado de daño estructural cerebral puedan contribuir a estos resultados contradictorios.

En este sentido, la EM es una enfermedad con un amplio sustrato neuropatológico en la que, además de producirse atrofia y cambios funcionales, también son habituales las lesiones en la sustancia blanca y en la sustancia gris (tanto corticales como subcorticales), que se han relacionado con alteraciones de la CF entre regiones cerebrales y con el estado cognitivo [29,30]. Por eso, pensamos que una completa explicación del deterioro cognitivo en la EM debe contemplar todas estas medidas.

Para finalizar, debemos considerar varias limitaciones de nuestro estudio. En primer lugar, existen diferencias entre pacientes y sujetos control en edad, escolaridad y CI manipulativo, que representan variables que se relacionan con el estado cognitivo, así como con la pérdida de tejido cerebral y las alteraciones de la CF en las redes cerebrales. En este sentido, la realización de estudios con muestras experimentales más homogéneas resultaría conveniente para obtener conclusiones más sólidas. En segundo lugar, no hemos podido analizar la contribución de las lesiones en la sustancia gris sobre el rendimiento cognitivo, debido a que no disponemos de las secuencias de resonancia magnética apropiadas para identificarlas.

En conclusión, el presente estudio muestra una clara relación entre bajos rendimientos en tareas de memoria episódica/declarativa en pacientes con EM y alteraciones estructurales y funcionales observadas en el hipocampo, estructura anatómica relacionada con este tipo de memoria.

\section{Bibliografía}

1. Chiaravalloti ND, DeLuca J. Cognitive impairment in multiple sclerosis. Lancet Neurol 2008; 7: 1139-51.

2. Bobholz JA, Gremley S. Multiple sclerosis and other desmyelinating disorders. In Schoenberg MR, Scott JG, eds. The little black book of neuropsychology: a syndrome-based approach. New York: Springer; 2011. p. 647-61.

3. Olascoaga J. Calidad de vida y esclerosis múltiple. Rev Neurol 2010; 51: 279-88.

4. Denney DR, Sworowski LA, Lynch SG. Cognitive impairment in three subtypes of multiple sclerosis. Arch Clin Neuropsychol 2005; 20: 967-81.

5. Andreu-Català M, Pascual Lozano AM, Bueno-Cayo A, Boscá-Blasco I, Coret-Ferrer F, Casanova-Estruch B. Afectación de las funciones cognitivas en la esclerosis múltiple secundaria progresiva. Rev Neurol 2008; 46: 664-6.

6. Sicotte NL, Kern KC, Giesser BS, Arshanapalli A, Schultz A, Montag M, et al. Regional hippocampal atrophy in multiple sclerosis. Brain 2008; 131: 1134-41.

7. Benedict RH, Cookfair D, Gavett R, Gunther M, Munschauer F. Validity of the minimal assessment of cognitive function in
Figura 2. a) Resultados de las diferencias de conectividad funcional entre el grupo de los pacientes y el grupo control: reducción de la conectividad funcional del hipocampo izquierdo con diferentes regiones cerebrales en el grupo de pacientes; b) Correlación entre la $Z$ visual (Zvi) y la conectividad funcional del hipocampo izquierdo con diferentes regiones observadas en el grupo de pacientes. Para el análisis se utilizó una $p<0,001$ corregida en el clúster mediante simulación de Monte Carlo, utilizando el programa Alphasim de AFNI, y con covarianza de los análisis por edad y sexo.

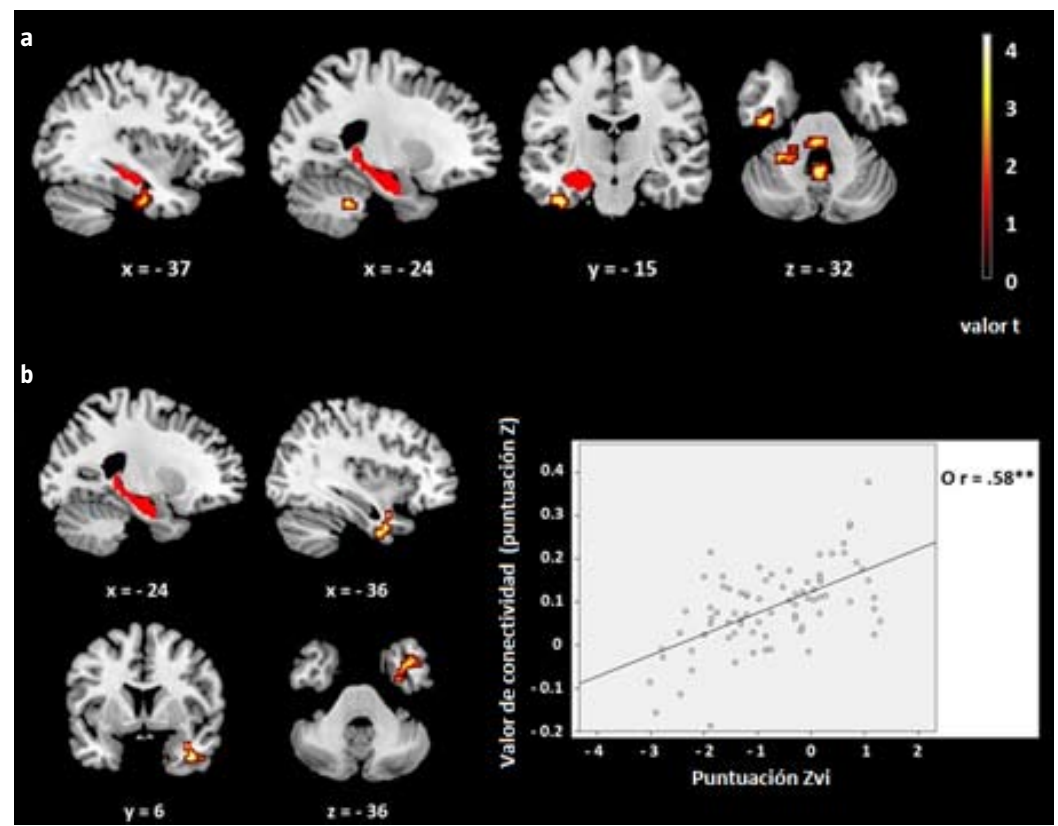

multiple sclerosis (MACFIMS). J Int Neuropsychol Soc 2006; 12: 549-58.

8. Rao SM, Leo GJ, Bernardin L, Unverzagt F. Cognitive dysfunction in multiple sclerosis. I. Frequency, patterns, and prediction. Neurology 1991; 41: 685-91.

9. Longoni G, Rocca MA, Pagani E, Riccitelli GC, Colombo B, Falini A, et al. Deficits in memory and visuospatial learning correlate with regional hippocampal atrophy in MS. Brain Struct Funct 2015; 220: 435-44.

10. Filippi M, Rocca MA, Benedict RH, DeLuca J, Geurts JJ, Rombouts SA, et al. The contribution of MRI in assessing cognitive impairment in multiple sclerosis. Neurology 2010; 75: 2121-8.

11. Orozco-Giménez C, Pastor-Pons E, Meersmans SánchezJofré M, Verdejo-García A, Pérez-García M. Relación entre los tests de memoria y reflexología del hipocampo. Rev Neurol 2005; 40: 129-34.

12. Anderson VM, Fisniku LK, Khaleeli Z, Summers MM, Penny SA, Altmann DR, et al. Hippocampal atrophy in relapsing-remitting and primary progressive MS: a comparative study. Mult Scler 2010; 16: 1083-90.

13. Roosendaal SD, Hulst HE, Vrenken H, Feenstra HE, Castelijns JA. Structural and functional hippocampal changes in multiple sclerosis patients with intact memory function. Radiology 2010; 255: 595-604.

14. Cruz-Gómez AJ, Ventura-Campos N, Belenguer A, Ávila C, Forn $\mathrm{C}$. The link between resting-state functional connectivity and cognition in MS patients. Mult Scler 2014; 20: 338-48.

15. Hulst HE, Schoonheim MM, Van Geest Q, Uitdehaag BM, Barkhof F, Geurts JJ. Memory impairment in multiple sclerosis: 
relevance of hippocampal activation and hippocampal connectivity. Mult Scler 2015; 21: 1705-12.

16. Polman $\mathrm{CH}$, Reingold SC, Edan G, Filippi M, Hartung HP Kappos L, et al. Diagnostic criteria for multiple sclerosis: 2005 revisions to the 'McDonald criteria'. Ann Neurol 2005; 58: 840-6.

17. Krupp LB, LaRocca NG, Muir-Nash J, Steinberg AD. The fatigue severity scale. Application to patients with multiple sclerosis and systemic lupus erythematosus. Arch Neurol 1989; 46: 1121-3.

18. Bulbena A, Berrios G, Fernández de Larrinoa PP. Medición clínica en psiquiatría y psicología. Barcelona: Masson; 2000.

19. Rao SM; Cognitive Function Study Group of the National Multiple Sclerosis Society. A manual for the Brief Repeatable Battery of Neuropsychological Tests in multiple sclerosis Milwaukee, WI: Medical College of Wisconsin; 1990.

20. Sepulcre J, Vanotti S, Hernández R, Sandoval G, Cáceres F, Garcea O, et al. Cognitive impairment in patients with multiple sclerosis using the Brief Repeatable Battery-Neuropsychology test. Mult Scler 2006; 12: 187-95.

21. Forn C, Arnett P. Evaluación neuropsicológica en la esclerosis múltiple. Rev Neurol 2007; 44: 166-72.

22. Gelineau-Morel R, Tomassini V, Jenkinson M., JohansenBerg H, Matthews PM, Palace J. The effect of hypointense white matter lesions on automated gray matter segmentation in multiple sclerosis. Hum Brain Mapp 2012; 33: 2802-14.
23. Chao-Gan Y, Yu-Feng Z. DPARSF: a MATLAB toolbox for 'pipeline' data analysis of resting-state fMRI. Front Syst Neurosci 2010; 4: 13.

24. Maldjian JA, Laurienti PJ, Kraft RA, Burdette JH. An automated method for neuroanatomic and cytoarchitectonic atlas-based interrogation of fMRI data sets. Neuroimage 2003; 19 1233-9.

25. Cherbuin N, Anstey KJ, Reglade-Meslin C, Sachdev PS. In vivo hippocampal measurement and memory: a comparison of manual tracing and automated segmentation in a large community-based sample. PLoS One 2009; 4: e5265.

26. Suzuki WA. Declarative versus episodic: two theories put to the test. Neuron $2003 ; 38: 5-7$.

27. Mitchell KJ, Johnson MK. Source monitoring 15 years later: what have we learned from fMRI about the neural mechanisms of source memory? Psychol Bull 2009; 135: 638-77.

28. Schoonheim MM, Geurts JJ, Barkhof F. The limits of functional reorganization in multiple sclerosis. Neurology 2010; 74: 1246-7.

29. Messina S, Patti F. Gray matters in multiple sclerosis: cognitive impairment and structural MRI. Mult Scler Int 2014; 2014: 609694.

30. Rossi F, Giorgio A, Battaglini M, Stromillo ML, Portaccio E, Goretti B, et al. Relevance of brain lesion location to cognition in relapsing multiple sclerosis. PLoS One 2012; 7: e44826.

\section{Structural and functional changes of the hippocampus in patients with multiple sclerosis and their relationship with memory processes}

Introduction. One of the most prevalent cognitive deficits in multiple sclerosis (MS) patients relates to (both verbal and visual) information encoding, a process related the hippocampus.

Aim. To assess the relationship between information learning and long-term retention processes and hippocampal volume and functional connectivity (FC) in MS patients.

Subjects and methods. MS patients and a control group of healthy volunteers were assessed using the brief neuropsychological battery, which includes tests of verbal and visual memory. Gray matter volume was assessed through the voxel based morphometry technique, and a study of seed-based FC focused on the region of interest (hippocampus) was also conducted.

Results. Results revealed that, as compared to the control group, MS patients display gray matter atrophy at the left hippocampus gray matter and smaller FC between left hippocampus and brainstem, cerebellum, fusiform gyrus and superior temporal gyrus. Association analyses were performed between memory tests performance and both volumetric and FC changes. Results showed a positive correlation between gray matter hippocampal (bilateral) volume and verbal memory performance of the MS group. Further, in MS patients, a positive correlation between visual memory performance and FC between the left hippocampus and several temporal regions was also found.

Conclusions. The results of the present study reveal a relationship between structural and functional changes in the hippocampus of MS patients and their performance on verbal and visual memory tests.

Key words. Functional connectivity. Gray matter. Hippocampus. Memory. Multiple sclerosis. Volumetry. 